\title{
Relación entre el perfil facial mediante análisis de Arnett-Bergman y el elemento II de Andrews en estudiantes de una escuela profesional de odontología en Tacna
}

\author{
Relationship between facial profile through Arnett-Bergman analysis and \\ Andrews element II in students of a professional dental school in Tacna
}

\author{
Wender Williams Condori Quispe ${ }^{1 \mathrm{a}}$, Isabel del Rosario Ayca Castro ${ }^{2^{\mathrm{b}}}$ \\ ${ }^{1}$ Escuela Profesional de Odontología de la Universidad Nacional Jorge Basadre Grohmann. Tacna, Perú. \\ ${ }^{2}$ Escuela Profesional de Odontología de la Universidad Nacional Jorge Basadre Grohmann. Tacna, Perú. \\ ${ }^{a}$ Docente, Maestro en Odontoestomatologia. \\ ${ }^{\mathrm{b}}$ Docente, Maestro en Odontoestomatologia.
}

\begin{abstract}
Resumen
Este estudio se realizó en la Escuela de Odontología de la Universidad Nacional Jorge Basadre Grohmann de Tacna en el año 2017. Objetivo: determinar la relación del perfil facial mediante el análisis propuesto por Arnett - Bergman y el Elemento II de Andrews. Material y Métodos: El tipo de estudio es de campo y de nivel relacional, de corte transversal. Asimisno, es bivariable, ya que presenta el perfil facial con sus respectivos indicadores y subindicadores. La técnica e instrumento seleccionados fueron: observación directa y guía de observación a través de fotografías faciales. Instrumento aplicado a 57 estudiantes seleccionados. Conclusions: 1) El $48.4 \%$ de los estudiantes que no presentan correlación son de sexo femenino, y el $51.6 \%$ son del género masculino. 2) Según el análisis de Arnett - Bergman, se encontró que el $56 \%$ de los estudiantes corresponde a la clase I, el $29 \%$ de ellos se ubica en la clase II y el $14 \%$ en la clase III. 3), según el análisis del elemento II de Andrews el $54.4 \%$ no coincide con la vertical frontal y el $45.6 \%$ sí coincide. 4) Se concluye que existe relación entre ambos análisis faciales de ArnettBergman y el elemento II de Andrew, el 78.1 \% de estudiantes que tienen un perfil facial de Clase I según ArnettBergman con la coincidencia de la vertical frontal de Andrews.

Palabras clave: Análisis de Arnett Bergman, elemento II de Andrews, perfil facial.
\end{abstract}

\begin{abstract}
This study was carried out at the School of Dentistry, Jorge Basadre Grohmann National University, Tacna in 2017. Objective: to determine the relationship of the facial profile through the analysis proposed by Arnett-Bergman and Andrews Element II. Material and Methods: It is a field study and relational research level, as well as crosssectional. Also, it is bivariable, since it presents the facial profile with its respective indicators and subindicators. The technique and instrument selected were: direct observation and observation guide through facial photographs. The instrument was applied to 57 selected students. Conclusions: 1) $48.4 \%$ of the students who do not have a correlation are female, and $51.6 \%$ are male. 2) According to Arnett-Bergman's analysis, it was found that $56 \%$ of the students correspond to class I, $29 \%$ of them are in class II and $14 \%$ in class III. 3). According to the analysis of Andrews element II, $54.4 \%$ does not coincide with the vertical front and $45.6 \%$ does. 4) It is concluded that there is a relationship between both facial analyzes of Arnett - Bergman and Andrew's Element II, $78.1 \%$ of students who have a Class I facial profile according to Arnett - Bergman with the coincidence of Andrews frontal vertical.

Keywords: Arnett Bergman analysis, Andrews element II, facial profile.
\end{abstract}




\section{Introducción}

La interrelación de un buen diagnóstico con el tratamiento es la medida acertada para la atención de las alteraciones dentomaxilares (1). El estudio clínico facial, los modelos de estudio, la cefalometría y fundamentalmente las fotografías constituyen la piedra angular de un buen diagnóstico odontológico. Un examen prolijo del perfil facial y una puntual información del análisis de las radiografías cefalométricas laterales son esenciales para llegar a una conclusión diagnóstica segura (2).

Actualmente, tanto el aspecto corporal como el aspecto facial tienen un papel relevante en el estado psicológico de las personas. La ciencia médica que abarca la estética facial es la odontología a través de la especialidad de ortodoncia, la cual por medio de un adecuado diagnóstico, planificación y ejecución del tratamiento ortodóncico en base a movimientos biodinámicos puede intervenir en el cambio del tercio inferior de la cara, el cual es un punto de partida para la elaboración de diferentes tipos de análisis faciales desde los más sofisticados y extensos hasta los más simples y sencillo (3).

Es imprescindible considerar la estética facial; al respecto, existen estudios de análisis faciales, dentro de ellos se encuentran el de Arnett - Bergman y el elemento II de Andrews. Ambos estudios aportan datos y valores del perfil facial, sistemáticos y precisos con la cabeza en posición normal.

En nuestro contexto existe una variedad de razas, cuyas características faciales se deben estudiar como medida preventiva odontológica de alteraciones dentomaxilares.

\section{Material y método}

El presente estudio utilizó fotografías laterales en posición natural de la cabeza, con y sin sonrisa de los estudiantes seleccionados. La investigación se realizó durante los meses de agosto a diciembre del 2017. La población estuvo constituida por la totalidad de alumnos matriculados en la Escuela Profesional de Odontología durante el periodo académico 2017.

$\begin{array}{ccc}\text { Tabla 1. Perfil facial de los estudiantes de la } \\ \text { Escuela Profesional de Odontología de la } \\ \text { Universidad Nacional Jorge Basadre } \\ \text { Grohmann según análisis de Arnett - } \\ \text { Bergman } & & \\ \text { Análisis de Arnett-Bergman } & \text { Frecuencia } & \text { Porcentaje } \\ \text { Clase I } & 3 & 56.1 \\ \text { Clase II } & 1 & 29.8 \\ \text { Clase III } & \varepsilon & 14.0 \\ \text { Total } & 5 & 100.0\end{array}$

Fuente: Matriz de datos
En la tabla y gráfico 3 se observa los resultados del perfil facial de los estudiantes previa toma y análisis fotográfico y técnica de trazado de Arnett-Bergman; de ellos, el $56 \%$ se ubica en la clase I por que corresponden a los ángulos entre $165^{\circ}$ y $175^{\circ}$, lo cual nos indica una relación anteroposterior normal de los maxilares.

El $29 \%$ de ellos se ubica en la clase II, que corresponde a ángulos menores de $165^{\circ}$, esto representa el maxilar superior adelantando con respecto a la mandíbula.

Solo el $14 \%$ de los estudiantes se ubican en la clase III por tener perfil cóncavo; que corresponde a los ángulos mayores a $175^{\circ}$, lo cual representa el maxilar superior retrasado con respecto a la mandíbula.

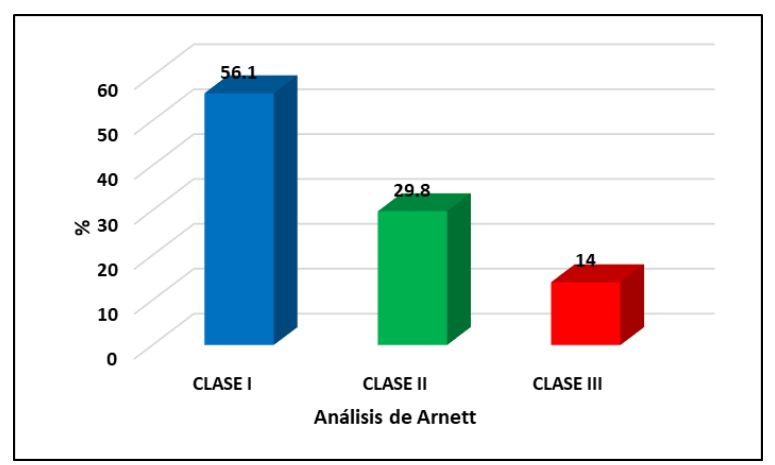

Gráfico 1. Análisis de Arnett. Fuente: Matriz de datos.

En la Tabla 2 se aprecia que, con respecto a la armonía facial, según el elemento II de Andrews, en el $54 \%$ de los estudiantes investigados no existe correlación entre las posiciones de las piezas dentarias del maxilar superior con la vertical frontal; es decir, que no hay coincidencia. Asimismo, en el $45.6 \%$ de los estudiantes, sí existe coincidencia.

\begin{tabular}{|c|c|c|}
\hline Análisis de Andrews-VF & Frecuencia & Porcentaje \\
\hline NO Coincide & 31 & 54.4 \\
\hline SÍ Coincide & 26 & 45.6 \\
\hline Total & 57 & 100 \\
\hline
\end{tabular}

Fuente: Matriz de datos 


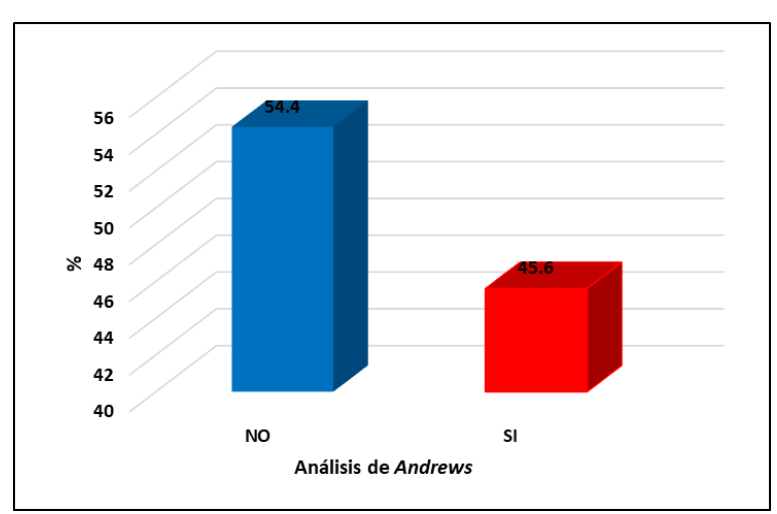

Gráfico 2. Análisis de Andrews.

Fuente: Matriz de datos

En la tabla 3 se observa que del $62.5 \%$ de estudiantes con perfil facial de clase I y III de Arnett - Bergman corresponde al sexo femenino y el $37.5 \%$ al sexo masculino. El $64.7 \%$ con perfil facial clase II de Arnett Bergman corresponde al sexo femenino y el $35.5 \%$ corresponde al sexo masculino.

Por lo tanto, no se relacionan entre sí las variables, ya que el análisis de Arnett - Bergman difiere según sexo.

\section{Tabla 3. Relación entre el análisis de Arnett - Bergman y sexo}

\begin{tabular}{|c|c|c|c|c|}
\hline \multirow{2}{*}{\multicolumn{2}{|c|}{ Arnet - Bergman }} & \multicolumn{2}{|c|}{ Sexo } & \multirow[b]{2}{*}{ Total } \\
\hline & & Masculino & Femenino & \\
\hline \multirow[t]{2}{*}{ Clase I } & $\mathrm{n}$ & 12 & 20 & 32 \\
\hline & $\%$ & 37.5 & 62.5 & 100.0 \\
\hline \multirow[t]{2}{*}{ Clase II } & $\mathrm{n}$ & 6 & 11 & 17 \\
\hline & $\%$ & 35.3 & 64.7 & 100.0 \\
\hline \multirow[t]{2}{*}{ Clase III } & $\mathrm{n}$ & 3 & 5 & 8 \\
\hline & $\%$ & 37.5 & 62.5 & 100.0 \\
\hline \multirow[t]{2}{*}{ Total } & $\mathrm{n}$ & 21 & 36 & 57 \\
\hline & $\%$ & 36.8 & 63.2 & 100.0 \\
\hline
\end{tabular}

$\begin{array}{lccc} & \text { Valor } & \text { df } & \begin{array}{l}\text { Significación asintótica } \\ \text { (bilateral) }\end{array} \\ \text { Chi cuadrado de Pearson } & .025^{\mathrm{a}} & 2 & .988 \\ \text { Razón de verosimilitud } & .025 & 2 & .988 \\ \text { Asociación lineal por lineal } & .004 & 1 & .953 \\ \text { n de casos válidos } & 57 & \\ \text { a. } 1 \text { casillas (16.7 \%) han esperado un recuento menor que 5. E } \\ \text { El recuento mínimo esperado es 2.95 }\end{array}$

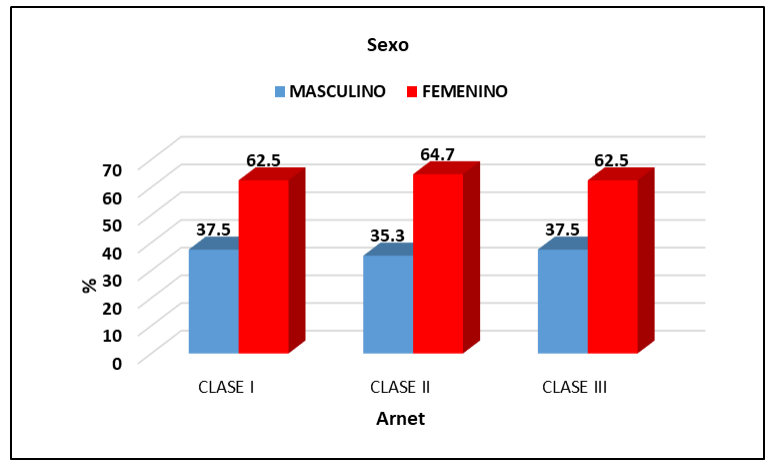

Gráfico 3. Análisis de Arnet

En la tabla y gráfico 4 se observa que el $80.8 \%$ estudiantes presentan correlaciones entre las posiciones de las piezas dentarias del maxilar superior con la vertical frontal, del cual el $63.2 \%$ representa al sexo femenino y el $19.2 \%$ al sexo masculino.

El $51.6 \%$ de estudiantes que no presentan correlación entre las posiciones de las piezas dentarias del maxilar superior con la vertical frontal son de sexo masculino, mientras que el $48.4 \%$ son de sexo femenino. Por lo tanto, se observa que sí existe relación entre sexo y el elemento II de Andrews.

\begin{tabular}{|c|c|c|c|c|}
\hline \multicolumn{5}{|c|}{ Elemento II de Andrews } \\
\hline & VF & Masculino & $\begin{array}{l}\text { Sexo } \\
\text { Femenino }\end{array}$ & Total \\
\hline \multirow[t]{2}{*}{ No coincide } & $\mathrm{n}$ & 16 & 15 & 31 \\
\hline & $\%$ & 51.6 & 48.4 & 100.0 \\
\hline \multirow{2}{*}{$\begin{array}{l}\mathrm{Si} \\
\text { coincide }\end{array}$} & $\mathrm{n}$ & 5 & 21 & 26 \\
\hline & $\%$ & 19.2 & 80.8 & 100.0 \\
\hline \multirow[t]{2}{*}{ Total } & $\mathrm{n}$ & 21 & 36 & 57 \\
\hline & $\%$ & 368 & 63.2 & 100.0 \\
\hline \multicolumn{5}{|c|}{$\mathrm{P}=0.012$ es significativa $\mathrm{p}<\alpha=0.05$} \\
\hline
\end{tabular}

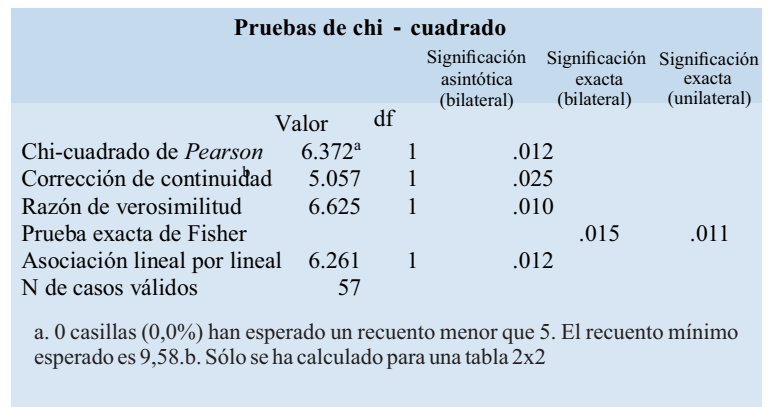




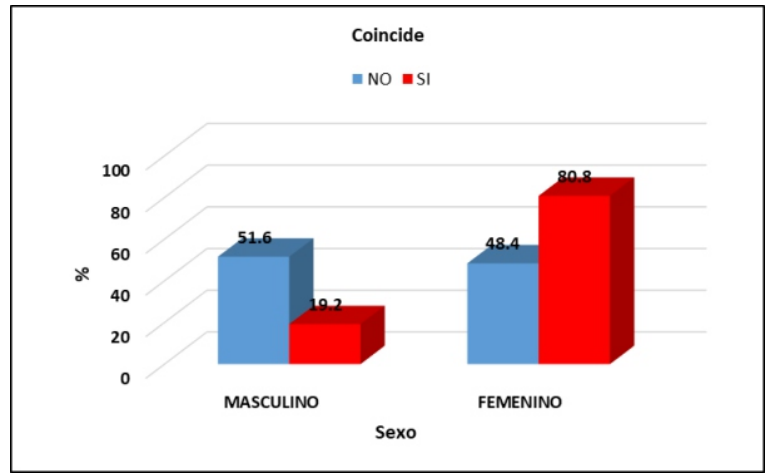

Gráfico 5.

Fuente: Matriz de datos

En la tabla 5 se observa que el $78.1 \%$ de estudiantes que tienen un perfil facial de clase I según el Análisis de Arnett-Bergman presentan una correlación con el análisis del elemento II de Andrews que sí tienen coincidencia con la vertical frontal, frente al $21.9 \%$ que no muestra coincidencia con la vertical frontal.

El $87.5 \%$ de los estudiantes que poseen perfil de clase III según el Análisis de Arnett-Bergman y del $12.5 \%$ según el análisis del elemento II de Andrews que sí tienen coincidencia con la vertical frontal no muestran coincidencia. El $100 \%$ de estudiantes que poseen un perfil de clase II no muestra coincidencia por lo que se aprecia que existe relación directa.

\section{Tabla 5. Relación entre análisis de Arnett - Bergman y del elemento II de Andrews

\begin{tabular}{|c|c|c|c|c|}
\hline \multirow{2}{*}{\multicolumn{2}{|c|}{$\begin{array}{c}\text { Análisis de Arnett - } \\
\text { Bergman }\end{array}$}} & \multicolumn{2}{|c|}{ Elemento II de Andrews } & \multirow[t]{2}{*}{ Total } \\
\hline & & & Sí coincide & \\
\hline Clase I & $n_{0}^{n}$ & 79 & 25 & $\begin{array}{l}32 \\
1000\end{array}$ \\
\hline Clase II & $\begin{array}{l}\text { no } \\
\mathrm{n}\end{array}$ & 17 & 0 & 17 \\
\hline Clase III & $\mathrm{n}$ & $\begin{array}{l}100.0 \\
7\end{array}$ & $0_{1}^{0}$ & 8 \\
\hline Total & $\begin{array}{l}\% \\
\text { n } \\
\%\end{array}$ & $\begin{array}{l}81.3 \\
31.4\end{array}$ & $\begin{array}{l}2.3 \\
26 \\
45.6\end{array}$ & $\begin{array}{l}100.0 \\
57 \\
100.0\end{array}$ \\
\hline \multicolumn{5}{|c|}{ iva $\mathrm{p}<\alpha=0.05$} \\
\hline
\end{tabular}

\begin{tabular}{lrccc} 
& \multicolumn{3}{c}{ Pruebas de chi-cuadrado } \\
& \multicolumn{4}{c}{$\begin{array}{c}\text { Significación asintótica } \\
\text { (bilateral) }\end{array}$} \\
Chi-cuadrado de Pearson & $\begin{array}{l}\text { Valor } \\
\text { Razón de verosimilitud }\end{array}$ & df & & .000 \\
Asociación lineal por lineal & 22.931 & 2 & .000 \\
N de casos válidos & 22.570 & 1 & .000 \\
a. 2 casillas (33,3\%) han esperado un recuento menor que 5. El recuento \\
mínimo esperado es 3,65.
\end{tabular}

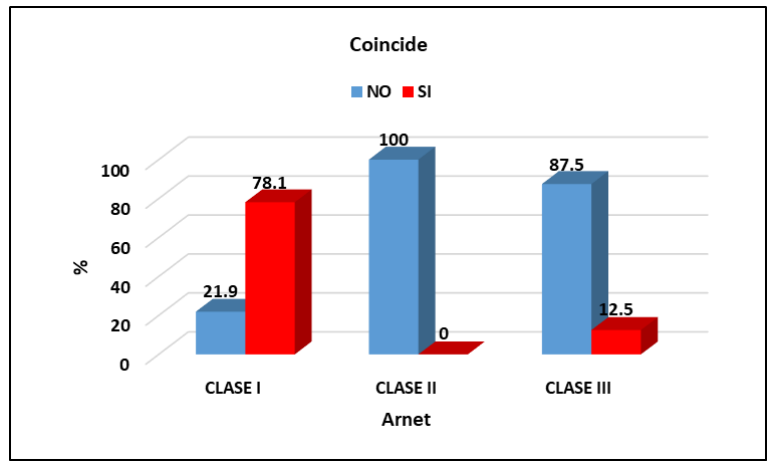

Gráfico 5. Análisis de Arnet

Fuente: Matriz de datos

\section{Discusión y comentarios}

En atención a los resultados, sobre el indicador edad, se sostiene que en odontología como en cualquier otra carrera, los estudiantes en su mayoría pertenecen al ciclo vital adolescentes y adultos jóvenes, ya que presentan edades correspondientes al rango de 17 a 31 años, edad en la que la constitución física y mental de las personas están en franco progreso vital, dando así la posibilidad de un mejor rendimiento, en este caso, académico (4).

En lo relacionado al género, el mayor porcentaje corresponde al género femenino, siendo la proporción de 6 mujeres a 3 varones (5). Por lo general, se puede comprobar que la oportunidad de estudio superior tanto para varones como para mujeres ha cambiado desde hace varias décadas, no siendo ajeno a esto, el ingreso del sexo femenino a las diferentes carreras profesionales (6).

En relación a los resultados encontrados según sexo, se observa que el $63.2 \%$ corresponde al sexo femenino y el $36.8 \%$ corresponde al sexo masculino, coincidiendo con trabajos realizados tanto por Pomari y Lima (2008) como de Novoa y Trujillo (2017)

En relación al tema investigado "perfil facial", según el análisis propuesto por Arnet - Bergman, se obtuvo como resultado que el $56.1 \%$ de estudiantes presentan clase I superando a la clase II (29.8 \%). Aquí coincidimos con los resultados tanto de Pomari y Lima (2008) como de Novoa y Trujillo (2017) que no encontraron diferencias entre las posiciones por presentar ángulos menores de $165^{\circ}(8)$.

De acuerdo con el perfil facial de los estudiantes investigados según el análisis del elemento II de Andrews, el 54.4\% no coincide con las posiciones de las piezas dentarias del maxilar superior con la vertical frontal, a diferencia del $45.6 \%$ que sí coincide con la vertical frontal, lo que concuerda con las investigaciones de Vallejos y Trujillo (9).

Con respecto a la correlación entre los análisis faciales Arnett - Bergman y el elemento II de Andrews, sí existe correlación entre ambas variables de estudio 
discrepando con Vásquez y Trujillo (2017) y coincidiendo con Vallejos y Trujillo (2015).

$\mathrm{Al}$ respecto, se puede mencionar que la según los autores como Proffit y Alarcón (10), no existe un patrón étnico sino una variedad de razas étnicas y anatómicas entre ambas poblaciones.

\section{Conclusiones}

- Se concluye que el $48.4 \%$ de los estudiantes que no presentan correlaciones son de sexo femenino. Asimismo, el $51.6 \%$, son del género masculino.
- Según el análisis de Arnett - Bergman, se encontró que el $56 \%$ de los estudiantes corresponde a la clase I; el 29\% se ubica en la clase II y el $14 \%$, en la clase III.

- Según el análisis del elemento II de Andrews, el $54 \%$ no coincide y el $45.6 \%$ sí coincide con la vertical frontal respectivamente.

- Se concluye que existe relación entre ambos análisis faciales de Arnett-Bergman en Clase I y el elemento II de Andrews que coincide con la vertical frontal en un $78.1 \%$, lo cual estadísticamente es muy significativa.

\section{Referencias Bibliográficas}

1. Canut J. A. "Ortodoncia Clínica Y Terapéutica" Barcelona, España: Ed. Masson-Salvat Odontología. 2000

2. Vela H., Arturo. Diagnóstico precoz de las maloclusiones esqueléticas y dentales en la infancia. Boletín de la sociedad vasco-navarra de pediatría. Vitoria, España. 2004.

3. Mendoza C, Marco A, "Análisis Facial en Ortodoncia" Residente en la Especialización de Ortodoncia UPCH Ed. KIRU. Vol. I. (1):3. 2004.

4. Panadent Guide. Golden Proportion Instructions. California, Estados Unidos 2008.

5. García, Eugenio.; Momose, Tami.; Mongruel, Osnara.; Gomes, Joao. Aplicación clínica de los parámetros estéticos en odontología restauradora.
Acta Odontológica Venezolana, Caracas, 20019; $47(1)$.

6. Saadia, M.; Ahlin, J. "Atlas de Ortopedia Dentofacial durante el crecimiento". Ed. Espaxs. Barcelona- España. 2000.

7. Simoes, Wilma A. Ortopedia Funcional de los Maxilares. Ed. Isaro. Caracas- Venezuela. 1988

8. Guevara M, Herrera C, Apumayta D. Análisis de Powell, de Arnett y Bergman. Año de la Unión Nacional Ante la Crisis Externa. 2009.

9. Gonzales-Ulloa M, Flores Es. (s.f.). Senility of the face; Basic study to undersatand its causes and effects. Plast reconst Surg. 1965.

10. Watkins P, Lubit E. Profile changes in the nowgrowing black patients following extractions mechano therapy. Journal of ortjodontics and dento facial orthopedics, $95-1992$.

\section{Correspondencia:}

Wender_cq@hotmail.com
Fecha de recepción : 14 de julio de 2019

Fecha de aceptación : 10 de noviembre 2019 